\title{
INVARIANT LINEAR FUNCTIONS ${ }^{1}$
}

\author{
BY \\ ROBERT J. SILVERMAN
}

1. Introduction. There have been many generalizations of the original Hahn-Banach extension theorem on the extension of a real-valued distributive function defined on a linear subspace of a real linear space where the extension is bounded from above by a positive-homogeneous, subadditive function. The generalizations have taken two forms. One is to add the requirement that the extensions be invariant with respect to a collection of operators defined on the domain space. Banach proved such a theorem for commutative groups [3]( $\left.{ }^{2}\right)$, Morse and Agnew for solvable groups [14]. The second type of generalization is to remove the restriction that the range space be the real numbers and assume instead, for example, that the range space be a partially ordered linear space of a special kind $[1 ; 2 ; 5 ; 7 ; 12 ; 15 ; 16]$.

Another extension problem closely related to Hahn-Banach extensions is that of extending a monotone distributive function defined on a subspace of an ordered linear space and whose range is an ordered linear space $[5 ; 9 ; 10$; $11 ; 13]$. The purpose of this paper is to consolidate the generalizations of the Hahn-Banach Theorem, relate these to invariant monotone extension theorems, and further extend the generalizations where there are minimal restrictions placed on the range space. This reduces the problem to a characterization of abstract semigroups.

2. Background material. The purpose of this section is to introduce without proofs some necessary background material. A knowledge of the basic concepts of linear spaces, groups, semigroups, and partially ordered systems is presumed. Unless otherwise noted, all linear spaces have the real numbers as the scalar field.

Definition 1. A linear space (LS) $V$ is an ordered linear space (OLS) if there exists a binary relationship, $\geqq$ (greater than or equal to), such that if $x, y, z$ are in $V$ and $t$ is any nonnegative real number, then (a) $x \geqq x$, (b) if $x \geqq y$ and $y \geqq z$, then $x \geqq z$, (c) if $x \geqq y$, then $t x \geqq t y$, (d) if $x \geqq y$, then $x+z \geqq y+z$.

Definition 2. A nonempty subset $C$ of a LS $V$ is a cone if $x, y$ in $C$ and $t$, a nonnegative real number, imply $x+y$, and $t x$ are in $C$. $C$ is sharp (SC) if $x$ and $-x$ in $C$ imply $x=0$.

Received by the editors March 11, 1955.

(1) This paper is essentially a portion of author's doctoral dissertation Invariant extensions of linear operators, University of Illinois, June, 1952. The author is deeply indebted to M. M. Day for his direction in the preparation of this work.

(2) The numbers in sauare brackets indicate references listed at the end of this paper. 
A. (1) A cone $C$ in a LS $V$ determines an ordering which makes $V$ an OLS: $x \geqq y$ if and only if $x-y$ is in $C$. (2) $C$, the set of elements greater than or equal to 0 , in an OLS $V$ is a cone. The order determined from $C$ is the original ordering (3) Cone $C$ is sharp if and only if $x \geqq y \geqq z$ imply $x=y$.

The definitions of upper bound (ub), lower bound (lb), least upper bound (sup), greatest lower bound (inf) of a subset of a partially ordered system are assumed, as is the concept of a directed system.

Definition 3. Given a directed system $Q$ with order indicated by $>$, and given a function $G$ from $Q$ to an OLS $V$, then the limit superior (lim sup) of $G$ and the limit inferior (lim inf) of $G$, if they exist, are defined as follows:

$$
\begin{aligned}
& \limsup _{Q \text { in } Q} G(q)=\inf _{Q \text { in } Q}\left(\sup _{q^{\prime}>Q} G\left(q^{\prime}\right)\right), \\
& \liminf _{Q \text { in } Q} G(q)=\sup _{Q}\left(\inf _{q^{\prime}>Q} G\left(q^{\prime}\right)\right) .
\end{aligned}
$$

A collection of properties associated with the above definitions follows.

$B$. The sup and inf of a subset of a partially ordered system are unique if they exist. If sup $U$ exists, $\sup (t U)=t$ sup $U$, where $U \subseteq V$, an OLS, and $t$ is a nonnegative real number. $(t U=\{t u: u$ in $U\}$.) $\sup (-U)=-\inf U$. $(-U=-1 U$.)

$$
\lim _{Q} \sup _{\operatorname{in} Q} G(q)=-\lim \inf (-G(q)) . \sup _{t \text { in } T}(G(t)+H(t)) \leqq \sup G(t)+\sup H(t),
$$

where $T$ is any index set, $G$ and $H$ are functions from $T$ to $V$, an OLS, and all the sups. exist. The preceeding inequality is true if $T$ is a directed system and sup is replaced by lim sup. sup $(U+W)=\sup U+\sup W$ and inf $(U+W)$ $=\inf U+\inf W$, where $U, W$ are subsets of $V$ an OLS, $(U+W=\{u+w$ : $u$ in $U, w$ in $W\}$ ), where it is assumed that $\sup U$, sup $W$, exist in the first equality and inf $U$, and inf $W$ exist in the second equality. If $W \subseteq U \subseteq V$, a partially ordered system, then sup $U \geqq \sup W$ and inf $W \geqq \inf U$, assuming the appropriate sups and infs exist.

DEFINITION 4. A function $m$ from an OLS $X$ into an OLS $Y$ is monotone if. $x \geqq x^{\prime}$ implies $m(x) \geqq m\left(x^{\prime}\right)$. $m$ is nonnegative if $x \geqq 0$ implies $m(x) \geqq 0$.

C. If $m$ is distributive, $m$ is nonnegative if and only if $m$ is monotone.

The main theorem of this introductory section will now be stated. Certain portions of it can be found in $[1 ; 2 ; 9 ; 10 ; 11 ; 13]$. However the organization and the generalized form are due to M. M. Day [5].

D. Consider an OLS $V$, then statements $1,2,3$, and 4 are equivalent and they imply 5 .

1. $V$ has the least upper bound property (LUBP). That is, every set of elements $A$ in $V$ with an upper bound has a least upper bound.

2. Given sets $A$ and $B$ in $V$ such that $a \geqq b$, for every $a$ in $A$ and $b$ in $B$, then there exists $v$ in $V$ such that $a \geqq v \geqq b$ for every $a$ in $A$ and $b$ in $B$. 
3. $V$ has the monotone extension property (MEP). That is, if $X$ is a subspace of and OLS $Y$ with cone $C$, such that (a) the cone in $X$ is $X \cap C$, (b) for all $y$ in $Y,(y+X) \cap C \neq \varnothing$ if and only if $(-y+X) \cap C \neq \varnothing$, then every monotone distributive function $f$ from $X$ to $V$ has a monotone, distributive extension $F$ from $Y$ to $V$.

4. $V$ has the monotone projection property (MPP). That is, if $X$ is replaced by $V$ in statement 3 above, there exists a monotone, distributive function $P$ from $Y$ onto $V$ such that $P(v)=v$ for every $v$ in $V$.

5. $V$ has the Hahn-Banach extension property (HBEP). That is, if (a) $X$ is a subspace of a linear space $Y$, (b) $p$ is a positive-homogeneous subadditive function from $Y$ to $V$, (c) $f$ is a distributive function from $X$ to $V$ such that $f(x) \leqq p(x)$ for every $x$ in $X$, then there exists a distributive extension $F$ of $f$ from $Y$ to $V$ such that $F(y) \leqq p(y)$ for every $y$ in $Y$.

3. Extension theorems. In the next two sections theorems will be proved about the class of semigroups which permit invariant monotone extensions. This class is identical with the class of semigroups which permit invariant Hahn-Banach extensions. This class in turn is contained in the class of semigroups which have invariant means definable on the associated Banach spaces of bounded real-valued functions. All semigroups which are known to have invariant means are shown to have the monotone extension property. It is conjectured that all the above classes coincide.

Definition 1. A representation of a semigroup (S) is defined in this paper to be a homomorphism or an antihomomorphism of (S) into the space of distributive operators of a vector space (or the image of $\mathbb{B}$ under such a map).

Definition 2. Consider (1) a linear space $Y$, (2) $X$, a subspace of $Y$, (3) an OLS $V$ with sharp positive cone $K$ and such that $V$ has the LUBP, (4) $G$, a semigroup of operators on $Y$, (5) $p$, a positive-homogeneous subadditive function from $Y$ to $V$ such that $p(g y) \leqq p(y)$ for all $y$ in $Y$ and $g$ in $G$, (6) $f$, a distributive function from $X$ to $V$ such that $f(g x)=f(x)$ and $f(x) \leqq p(x)$ for all $x$ in $X$ and $g$ in $G$. Then the collection $[Y, X, V, K, p, f, G]$ is said to have the Hahn-Banach extension property (HBEP) if there exists a distributive extension $F$ of $f$ defined from all of $Y$ to $V$ such that $F(y) \leqq p(y)$ and $F(g y)=F(y)$ for all $y$ in $Y$ and $g$ in $G$.

Definition 3. An abstract semigroup (5) has the Hahn-Banach extension property (HBEP) if every collection $[Y, X, V, K, p, f, G]$ as in Definition 2, subject to the additional restriction that $G$ is a representation of $(S)$, has the HBEP.

Definition 4. Consider (1) an OLS $Y$ with cone $C$, (2) $X$, an ordered linear subspace of $Y$ with ordering induced by the order in $Y$ such that $y+X$ meets $C$ for every $y$ in $Y$, (3) $G$, a semigroup of operators on $Y$ such that $g z$ is in $C$ and $g x$ is in $X$ for all $z$ in $C, x$ in $X$, and $g$ in $G$, (4) $V$, an OLS with sharp cone $K$ and such that $V$ has the LUBP, (5) $f$, a monotone, distributive function from $X$ to $V$ such that $f(g x)=f(x)$ for all $x$ in $X$ and $g$ in $G$. Then the 
collection $[Y, X, C, V, K, f, G]$ has the monotone extension property (MEP) if there exists a monotone distributive extension $F$ of $f$ from all of $Y$ to $V$ such that $F(g y)=F(y)$ for all $y$ in $Y$ and $g$ in $G$.

DEFINITION 5. An abstract semigroup (\$) has the monotone extension property (MEP) if every collection $[Y, X, C, V, K, f, G]$ as in Definition 4 , subject to the additional restriction that $G$ is a representation of $B$, has the MEP.

A few comments are in order. The condition in Definitions 2 and 4 that $K$ be a sharp cone and the condition in Definition 4 that $y+X$ meet $C$ for all $y$ in $Y$ are more restrictive than the corresponding conditions in the main theorem, D, of $\S 2$, but they appeared necessary in the proof of a few of the theorems in this section. Perhaps alternative proofs or modifications of the present proofs will eliminate the need for these conditions. A slightly weaker version of a portion of the main theorem in $\$ 2$ is that the group consisting only of the identity has the HBEP and the MEP.

It will be possible by virtue of the next theorem to restrict attention to semigroups with the MEP. The proof of the first part of the theorem is similar to the proof that 3 implies 5 in Theorem D, $\$ 2,[5]$.

THEOREM 1. A semigroup, (\$), has the MEP if and only if $\$$ h has the HBEP

Proof. Assume (B) has the MEP. (1) Consider $[Y, X, V, K, p, f, G]$ as in Definition 2, subject to the condition that $G$ is a representation of $\$ S$.

(2) Define the vector space $Y^{\prime}=\{(v, y): v$ in $V, y$ in $Y\}$, where addition and multiplication are defined co-ordinate wise. Define $C^{\prime}=\{(v, y): v \geqq p(y)\}$. It is a straightforward verification that $C^{\prime}$ is a cone in $Y^{\prime}$ and thus determines an order which makes $Y^{\prime}$ an OLS.

(3) Define the subspace $X^{\prime}$ of $Y^{\prime}$ :

$$
X^{\prime}=\{(v, x): v \text { in } V, x \text { in } X\} .
$$

$y^{\prime}+X^{\prime}$ meets $C^{\prime}$ for every $y^{\prime}$ in $Y^{\prime}$. For consider $y^{\prime}=(v, y)$, and choose any $x$ in $X$. Let $v^{\prime}=-v+p(x)+p(y)$. Then $\left(v^{\prime}, x\right)$ is in $X^{\prime}$ and $v^{\prime}+v=p(x)$ $+p(y) \geqq p(x+y)$. Hence $\left(v^{\prime}, x\right)+(v, y)=\left(v^{\prime}+v, x+y\right)$ is in $C^{\prime}$.

(4). Define the representation $G^{\prime}=(1, G)$ of $B$, where $(1, g)(v, y)=(v, g y)$. Each $g^{\prime}$ in $G^{\prime}$ clearly maps $X^{\prime}$ into itself. Each $g^{\prime}$ also maps $C^{\prime}$ into itself. This follows since if $(v, y)$ is in $C^{\prime}$, then $v \geqq p(y) \geqq p(g y)$ for all $g$ in $G$.

(5) Define the function $f^{\prime}$ from $X^{\prime}$ to $V: f^{\prime}(v, x)=v-f(x)$. Straightforward computation shows that $f^{\prime}$ is monotone, distributive, and fixed with respect to every $g^{\prime}$ in $G^{\prime}$. Thus by virtue of the assumption that \& has the MEP, there exists a monotone distributive extension $F^{\prime}$ of $f^{\prime}$ to all of $Y^{\prime}$ which is invariant with respect to every $g^{\prime}$ in $G^{\prime}$.

(6) Define $F$ from $Y$ to $V: F(y)=-F^{\prime}(0, y) . F$ is distributive and an extension of $f$, trivially. Also, $F(g y)=F(y)$ for all $y$ in $Y$ and $g$ in $G$. All that remains to be verified is that $F(y) \leqq p(y)$ for all $y$ in $Y$.

$F^{\prime}(p(y), y)=p(y)-F(y) . F^{\prime}$ is monotone and $(p(y), y)$ is in $C^{\prime}$, since 
$p(y) \geqq p(y)$. Hence, $p(y)-F(y)$ is in $K$; that is $F(y) \leqq p(y)$. Thus $(s)$ has the HBEP.

To prove the other part of the theorem, assume \& has the HBEP. (1) Take $\{Y, X, C, V, K, f, G\}$ as in Definition 4 , subject to the additional condition that $G$ is a representation of $(B)$.

(2) Define the function $p$ from $Y$ to $V: p(y)=\inf \{f(x): x$ in $X, x \geqq y\}$, for every $y$ in $Y$. $p$ is defined since $y+X$ meets $C$ for each $y$ and $V$ has the LUBP. Hence the inf exists and is unique.

(3) $p$ is positive-homogeneous since $f$ is homogeneous, and the inf is positive-homogeneous. The function $p$ is also subadditive for

$$
\begin{aligned}
p\left(y_{1}+y_{2}\right) & \leqq \inf \left\{f\left(x_{1}+x_{2}\right): x_{1}, x_{2} \text { in } X, x_{1} \geqq y_{1}, x_{2} \geqq y_{2}\right\} \\
& =\inf \left\{f\left(x_{1}\right): x_{1} \text { in } X, x_{1} \geqq y_{1}\right\}+\inf \left\{f\left(x_{2}\right): x_{2} \text { in } X, x_{2} \geqq y_{2}\right\} .
\end{aligned}
$$

The inequality holds since $x_{1}+x_{2} \geqq y_{1}+y_{2}$, and if set $A$ contains $B$ as a subset, then $\inf A \leqq \inf B$ (if the infs exist). The equality holds since the inf of the vector sum of two subsets of a vector space is the sum of the infs (if the infs exist). Hence $p$ is subadditive.

(4) $p(g y) \leqq p(y)$ for every $y$ in $Y$ and $g$ in $G$. To prove this, observe that if $x \geqq y$, then $g x \geqq g y$, and $\{g x: x$ in $X, g x \geqq g y\}$ is contained in $\{x: x$ in $X$, $x \geqq g y\}$. Therefore it follows that

$$
\begin{aligned}
p(y) & =\inf \{f(x): x \text { in } X, x \geqq y\} \geqq \inf \{f(g x)=f(x): g x \text { in } X, g x \geqq g y\} \\
& \geqq \inf \{f(x): x \text { in } X, x \geqq g y\}=p(g y) .
\end{aligned}
$$

(5) Thus, since $(\$)$ has the HBEP, there exists an invariant distributive extension $F$ of $f$ such that $F(y) \leqq p(y)$ for all $y$ in $Y$.

(6) $F(y)$ is monotone: If $y \leqq 0$, then $F(y) \leqq p(y) \leqq f(0)=0$, and thus $F$ is monotone. Hence 85 has the $\operatorname{MEP}\left({ }^{3}\right)$.

Definition 6. (1) Let $\mathfrak{S}$ be a subsemigroup of a semigroup (5). Define for each $g$ in $\mathbb{B}$ the right coset of $\mathfrak{S}, \mathfrak{S} g=\{g\} \cup\{h g: h$ in $H\}$, and similarly the left coset of $\mathfrak{S}, g \mathfrak{S}=\{g\} \cup\{g h: h$ in $\mathfrak{S}\}$. (2) A subsemigroup $\mathfrak{S}$ of $B$ is a commutator subsemigroup if for every $g, g^{\prime}$ in $\mathbb{S}, g g^{\prime}$ and $g^{\prime} g$ belong to the same left coset and the same right coset of $\mathfrak{S}$.

Consider a representation $G$ of $\$$ b. If the representation is a homomorphism, left (right) cosets of $\mathfrak{S}$ are mapped onto left (right) cosets of $H$, where $H$ is the image of $\mathfrak{S}$. However if the representation is an antihomomorphism, left (right) cosets are mapped onto right (left) cosets. In any case, cosets of a commutator subsemigroup are mapped onto cosets of a commutator subsemigroup. Observe that a function defined on a linear space which is invariant with respect to a subsemigroup, $H$, of a semigroup of operators is constant on right cosets of $H$.

(3) The referee suggested the proof of the second part of the theorem and the author is grateful. 
If $G$ is a group, the usual definition of a commutator subgroup satisfies the above definition. The proof of the next theorem makes use of a technique developed by Banach [3], and used by Morse and Agnew [14].

THEOREM 2. Let $\$$ be an abstract semigroup which contains a commutator subsemigroup $\mathfrak{S}$ with the $M E P$, then (5) has the $M E P$.

Proof. (1) Consider [ $Y, X, C, V, K, f, G]$ as in Definition 4 , where $G$ is a representation of $\mathbb{B}$. Restrict the representation $G$ of $\mathbb{B}$ to $H$ of $\mathfrak{S}$. Then since $\mathfrak{S}$ has the MEP, there exists a monotone distributive extension $F^{\prime}$ of $f$ to all of $Y$ such that $F^{\prime}(h y)=F^{\prime}(y)$ for every $h$ in $H$ and $y$ in $Y$.

(2) Define the function $p$ from $Y$ to $V$ :

$$
p(y)=\inf _{d \text { in } D} \sup _{g \text { in } G} S(d, g, y),
$$

where $(1 / N(d))\left(\sum_{g^{\prime} \text { ind }} F^{\prime}\left(g g^{\prime} y\right)\right)=S(d, g, y)$ for every $y$ in $Y$, where $D=\{d: d$ a finite subset of $G\}$, and $N(d)$ is the cardinality of $d$. Note that $S(d, g, x)=f(x)$ and $p(x)=f(x)$ for every $d, g$ and $x$.

(3) $p(y)$ exists for every $y$ in $Y$. Given a $y$, there exists an $x$ and an $x^{\prime}$ in $X$ such that $y+x$ and $-y+x^{\prime}$ are in $C$. Therefore, by the nonnegative property of $F^{\prime}$ and each $g$ in $G$, and the fact that $F^{\prime}(g x)=F^{\prime}(x)=f(x)$ for every $x$ in $X$ and every $g$ in $G$, it follows that $-f(x) \leqq S(d, g, y) \leqq f\left(x^{\prime}\right)$ for all $d$ and $g$. Hence, since $V$ has the LUBP, $p(y)$ exists.

(4) $p$ is a positive-homogeneous, subadditive function.

(a) $F^{\prime}\left(g_{1} g_{2} y\right)=F^{\prime}\left(g_{2} g_{1} y\right)$ for all $y$ in $Y$ and $g_{1}, g_{2}$ in $G$, for $g_{1} g_{2}$ and $g_{2} g_{1}$ are in the same right coset of $H$ since $\mathfrak{S}$ is a commutator subsemigroup of (5). The equality follows by virtue of the invariance of $F^{\prime}$ with respect to $H$.

(b) $\sup _{g \text { in } G} A\left(g^{\prime} g\right) \leqq \sup _{g \text { in } G} A(g)$ for any collection $\{A(g): g$ in $G\}$ contained in $V$ and any $g^{\prime}$ in $G$ provided sup $A(g)$ exists. This follows since $g^{\prime} G \subseteq G$. A similar statement with $g^{\prime} g$ replaced by $g g^{\prime}$ is also valid.

(c) Denote by $d_{1} d_{2}$ the collection $\left\{g_{1} g_{2}: g_{1}\right.$ in $d_{1}$ and $g_{2}$ in $\left.d_{2}\right\}$. Then

$$
\begin{aligned}
p\left(y_{1}+y_{2}\right) \leqq & \sup _{\sigma \text { in } G} S\left(d_{1} d_{2}, g, y_{1}+y_{2}\right) \\
= & \sup _{\sigma}\left(S\left(d_{1} d_{2}, g, y_{1}\right)+S\left(d_{1} d_{2}, g, y_{2}\right)\right) \\
\leqq & \sup _{\sigma} S\left(d_{1} d_{2}, g, y_{1}\right)+\sup _{\sigma} S\left(d_{1} d_{2}, g, y_{2}\right) \\
\leqq & \frac{1}{N\left(d_{2}\right)}\left(\sum_{g_{2}} \text { in } d_{2}\left(\sup _{\sigma} \frac{1}{N\left(d_{2}\right)}\right)\left(\sum_{\sigma_{1} \text { in } d_{1}} F^{\prime}\left(\left(g_{2} g\right) g_{1} y_{1}\right)\right)\right) \\
& +\frac{1}{N\left(d_{1}\right)}\left(\sum_{\theta_{1} \text { in } d_{1}}\left(\sup _{\sigma} \frac{1}{N\left(d_{2}\right)}\right)\left(\sum_{g_{2} \text { in } d_{2}} F^{\prime}\left(\left(g g_{1}\right) g_{2} y_{2}\right)\right)\right) \\
\leqq & \sup _{\sigma} S\left(d_{1}, g, y_{1}\right)+\sup _{\sigma} S\left(d_{2}, g, y_{2}\right)
\end{aligned}
$$


for every $d_{1}$ and $d_{2}$ in $D$. The last inequality follows in part from (b). The second to the last inequality follows in part from (a). Hence from the fact that $V$ has the LUBP,

$$
\begin{aligned}
p\left(y_{1}+y_{2}\right) & \leqq \inf _{d_{1}, d_{2} \text { in } D}\left(\sup _{\sigma} S\left(d_{1}, g, y_{1}\right)+\sup _{\sigma} S\left(d_{2}, g, y_{2}\right)\right) \\
& =\inf _{d_{1} \text { in } D} \sup _{\sigma} S\left(d_{1}, g, y_{1}\right)+\inf _{d_{2} \text { in } D} \sup _{\odot} S\left(d_{2}, g, y_{2}\right) \\
& =p\left(y_{1}\right)+p\left(y_{2}\right) .
\end{aligned}
$$

(5) Since $V$ has the LUBP, there exists an distributive extension $F$ of $f$ to all of $Y$ such that $F(y) \leqq p(y)$ for every $y$ in $Y$.

(6) $F$ is nonnegative. It is easily verified that $S$ is a nonnegative distributive function of $y$ for all arguments $g$ and $d$. This follows because a cone is a convex set, $F^{\prime}$ is a nonnegative function, and every $g$ in $G$ is a nonnegative operator. Consider $y$ in $C$.

$$
F(y) \geqq-p(-y)=-\inf _{d} \sup _{o}(-S(d, g, y))=\sup _{d} \inf _{g} S(d, g, y) \geqq 0 .
$$

(7) For every $g^{\prime}$ and every $y, F\left(g^{\prime} y\right)=F(y)$.

$$
\begin{aligned}
F\left(g^{\prime} y-y\right) & \leqq p\left(g^{\prime} y-y\right) \leqq \sup _{\odot} \frac{1}{N} \sum_{j=1}^{N} F^{\prime}\left(g g^{\prime}\left(g^{\prime} y-y\right)\right) \\
& =\sup _{0}\left[\frac{1}{N}\left(F^{\prime}\left(g g^{\prime N+1} y\right)-F^{\prime}\left(g g^{\prime} y\right)\right)\right] \leqq \frac{1}{N}\left(f(x)+f\left(x^{\prime}\right)\right),
\end{aligned}
$$

where $x$ and $x^{\prime}$ are fixed elements in $X$ as defined in (3)(a) of the proof. The inequality is true for all integers $N$. Hence $F\left(g^{\prime} y-y\right) \leqq 0$. By the same argument $F\left(y-g^{\prime} y\right) \leqq 0$. Hence, since $K$ is sharp, $F\left(g^{\prime} y\right)=F(y)$ for all $g^{\prime}$ and $y$.

THEOREM 3. An abstract commutative semigroup has the MEP.

Proof. The proof is almost identical with the proof of the last theorem, employing only the following two simplifications: $F^{\prime}$ is merely a monotone distributive extension of $f$, and commutivity eliminates the need for the argument in (4)(a) of the above theorem.

COROLlARY 1. Given a semigroup $G$ and a descending chain of subsemigroups of $G,\left\{G_{i}\right\}$, such that $G_{i+1}$ is a commutator subsemigroup of $G_{i}$ and $G=G_{1}$, then if there exists an element $G_{j}$ in the chain with the $M E P$, then $G$ has the MEP.

Definition 7. A semigroup $G$ is solvable if there exists a finite descending chain of subsemigroups $G_{i}, i=1, \cdots, N$, such that $G=G_{1}, G_{N}$ is commutative, and $G_{i+1}$ is a commutator subsemigroup of $G_{i}$. (If $G$ is a group this is equivalent to the definition of a solvable group.)

Corollary 2. A solvable semigroup (group) has the MEP. 
The next theorem is concerned with finite groups and is definitely false for finite semigroups. A counter example will be presented shortly.

THEOREM 4. A finite group has the MEP.

Proof. Let $G$ be a representation of the finite group on an OLS $Y$ with associated $C, X, V, K, f$ as in Definition 4 , then by the main theorem in $\S 2$, there exists a monotone distributive extension $F^{\prime}$ of $f$ to all of $Y$. Define $F$, the desired extension:

$$
F(y)=(1 / N) \sum_{0 \text { in } G} F^{\prime}(g y), \quad \text { where } N \text { is the order of } G .
$$

It is easily verified that $F$ is a monotone distributive extension of $f$. From the fact that multiplication by a fixed element of a group is merely a permutation of the group, it follows that $F\left(g^{\prime} y\right)=F(y)$ for all $g^{\prime}$ in $G$ and all $y$.

THEOREM 5. Given a semigroup (S) and given a collection of subsemigroups of $\mathfrak{B}, \mathfrak{D}=\{\mathfrak{b}\}$, with the properties $(\mathrm{a}) \bigcup_{\mathfrak{b i n} \mathbb{D}}(d)=\mathfrak{B},(\mathrm{b}) \mathfrak{D}$ forms a directed system under set inclusion, (c) each $\delta$ in $\mathfrak{D}$ has the $M E P$, then $\mathbb{B}$ has the MEP.

Proof. (1) Let $[Y, X, C, V, K, f, G]$ be defined as in Definition 4, where $G$ is a representation of (\$). The representation $G$ of $(S$ induces, by restriction, a representation $d$ of $\delta$ for every $\delta$ in $\mathfrak{D}$. Each representation satisfies the conditions in Definition 4. Hence since $\delta$ has the MEP, there exists a monotone distributive extension, $F_{d}$, of $F$ to all of $Y$ such that $F_{d}(g y)=F_{d}(y)$ for every $g$ in $d$ and $y$ in $Y$.

(2) Define $p(y)=\lim \sup _{d \text { in } D} F_{d}(y)=\inf _{d^{\prime} \text { in } D}\left(\sup _{d>d^{\prime}} F_{d}(y)\right)$, where $D$ is the collection of induced representations of the $\delta$ 's, $D$ clearly forms a directed system under set inclusion (order indicated by $>$ ). Note also that $\mathrm{U} d=G$.

(a) $p$ is well defined, (b) $p$ is positive-homogeneous and subadditive, and (c) $f$ is under $p$. By assumption, for each $y$ in $Y$ there exists an $x$ and $x^{\prime}$ in $X$ such that $y+x$ and $-y+x^{\prime}$ are in $C$. Thus $F_{d}(y+x)$ and $F_{d}\left(-y+x^{\prime}\right)$ are in $K$ for each $d$. From this it follows that $-f(x) \leqq F_{d}(y) \leqq f\left(x^{\prime}\right)$ for every $d$. Since $V$ has the LUBP, $p(y)$ exists and is unique for each $y$. From the properties of the lim sup it is clear that (b) holds. $f$ is under $p$. In fact, $f(x)=p(x)$ for every $x$ in $X$.

(3) Since $V$ has the LUBP, and hence the HBEP, it follows that there exists a distributive extension $F$ of $f$ to all of $Y$ such that $F(y) \leqq p(y)$ for all $y$.

(a) $F$ is monotone. Consider $y$ in $C$. Then

$$
\begin{aligned}
F(y) & \geqq-p(-y)=-\inf _{d^{\prime}}\left(\sup _{d>d^{\prime}} F_{d}(-y)\right) \\
& =\sup _{d^{\prime}}\left(\inf _{d>d^{\prime}} F_{d}(y)\right) \geqq 0, \text { since } F_{d}(y) \text { is in } K \text { for each } d .
\end{aligned}
$$


(b) The proof will be complete when it is shown that $F(g y)=F(y)$ for every $g$ in $G$ and $y$ in $Y$. It is asserted that

$$
p(g y-y)=p^{\prime}(g y-y), \text { where } p^{\prime}(g y-y)=\inf _{d^{\prime \prime} \text { in } D, 0 \text { in } d^{\prime \prime}}\left(\sup _{d>d^{\prime \prime}} F_{d}(g y-y)\right) .
$$

First, since the union of elements in $D$ cover $G$, such $d^{\prime \prime}$ 's exist for each $g$. In particular, for each $d^{\prime}$ in $D$ there exists a $d^{\prime \prime}$ in $D$ such that $g$ is in $d^{\prime \prime}$ and $d^{\prime \prime}>d^{\prime}$. Also it is noted that $\sup _{d>d^{\prime}} F_{d}(g y-y) \geqq \sup _{d>d^{\prime \prime}} F_{d}(g y-y)$ for $d^{\prime \prime}>d^{\prime}$. Hence taking the infimums with respect to the proper index sets it follows that $p^{\prime}(g y-y) \leqq p(g y-y)$. Since $\left\{d^{\prime \prime}: d^{\prime \prime}\right.$ in $D, g$ in $\left.d^{\prime \prime}\right\} \subseteq\left\{d^{\prime}: d^{\prime}\right.$ in $\left.D\right\}$, the reverse inequality follows. A more immediate proof could be given by making use of the theorem that replacing a directed system $D$ by a residual subset $D^{\prime \prime}$ of $D$ leaves lim sups unchanged.

Observe that $p^{\prime}(g y-y)=0$ for all $g$ and $y$. This follows since $F_{d}(g y-y)=0$ for $g$ in $d$. Hence $F(g y-y) \leqq 0$. Similarly $F(y-g y) \leqq 0$. Thus, by virtue of the sharpness of $K, F(y)=F(g y)$ for all $y$ and $g$.

The next theorem asserts that the MEP is preserved under group extensions.

THEOREM 6. Given an abstract group $(\$)$, a normal subgroup $\mathfrak{S}$ of $(\$)$, such that $\mathfrak{S}$ and the factor group $\mathfrak{M}=\mathbb{S} / \mathfrak{S}$ have the $M E P$, then $\mathbb{B}$ has the $M E P$.

Proof. (1) Consider $[Y, X, C, \dot{V}, K, f, G]$ as in Definition 4 , where $G$ is a representation of $(\mathcal{B}$. Restrict the representation $G$ of $(B)$ to $H$ of $\mathfrak{S}$. Since $\mathfrak{S}$ has the MEP, there exists a monotone distributive extension $F^{\prime}$ of $f$ to all of $Y$ with values in $V$ such that $F^{\prime}(h y)=F^{\prime}(y)$ for all $y$ in $Y$ and $h$ in $H$.

(2) Consider elements $g^{\prime}$ and $g^{\prime \prime}$ of $G$ which are in the same coset modulo $H$. Then $g^{\prime}=h g^{\prime \prime}$ for some $h$ in $H$. Hence $F^{\prime}\left(g^{\prime} y\right)=F^{\prime}\left(h g^{\prime \prime} y\right)=F^{\prime}\left(g^{\prime \prime} y\right)$ for all $y$ in $Y$. Therefore $F^{\prime}(y)$, considered as a function on $G$ for each $y$, is constant on cosets of $G$ modulo $H$.

(3) Define the function space $A$ to be the collection of all functions from $\mathfrak{M}$ to $V$. This clearly forms a vector space with respect to pointwise addition and multiplication by real scalars.

(4) Define the map $L$ from $Y$ to $A$ as follows: $L y=z$ in $A$, where $z(\mathfrak{m})$ $=F^{\prime}(g y)$ for any $g$ in the coset of $G$ modulo $H$ corresponding to $\mathrm{m}$. From (2) it is clear that $L$ is well defined. It is also easily checked that $L$ is a distributive function. Denote the image of $Y$ under the mapping $L$ by $Y^{\prime}$.

(5) Define the set $C^{\prime}$ in $Y^{\prime}: C^{\prime}=L C$, the image of the cone $C$ in $Y$. It can be verified easily that $C^{\prime}$ is a cone in $Y^{\prime}$. $C^{\prime}$ determines an ordering in $L Y$ which makes $Y^{\prime}$ an OLS, and $L$ a monotone function. It is noted that if $z$ is in $C^{\prime}$, then $z(\mathfrak{m})$ is in $K$ for every $\mathfrak{m}$ in $\mathfrak{M}$.

(6) Denote the image of $X$ with respect to $L$ by $X^{\prime} . q$ is in $X^{\prime}$ if and only if $q(\mathfrak{m})=f(x)$ for every $\mathfrak{m}$ in $\mathfrak{M}$ and for some $x$ in $X$.

(7) $z+X^{\prime}$ meets $C^{\prime}$ for every $z$ in $Y^{\prime}$. For given $z$ in $Y^{\prime}, z=L y$ for some 
$y$ in $Y$. Hence there exists $x$ in $X$ such that $y+x$ is in $C$. Hence $z+q=L y+L x$ $=L(y+x)$ is in $C^{\prime}$.

(8) Define the right regular representation $M$ of $\mathfrak{M}$ on $Y^{\prime}: m^{\prime} z(\mathfrak{m})$ $=\boldsymbol{z}\left(\mathfrak{m m}^{\prime}\right)$ for all $\mathfrak{m}$ in $\mathfrak{M}$ and $\boldsymbol{z}$ in $Y^{\prime} . m q=q$ for every $q$ in $X^{\prime}$ and $m$ in $M$. Also $m z$ is in $C^{\prime}$ for all $z$ in $C^{\prime}$ and $m$ in $M$. The last two statements are easily verified.

(9) Define $R$, a function from $X^{\prime}$ to $V: R(q)=f(x)$, for every $q$ in $X^{\prime}$ where $q=L x . R$ is (a) monotone (b) distributive, and (c) $R(m q)=R(q)$ for all $q$ in $X^{\prime}$ and $m$ in $M$. (c) follows immediately from (8). $R\left(t q+t^{\prime} q^{\prime}\right)=F^{\prime}\left(x^{\prime \prime}\right)$, where $L x^{\prime \prime}=t q+t^{\prime} q^{\prime}=t L x+t^{\prime} L x^{\prime}=t f(x)+t^{\prime} f^{\prime}\left(x^{\prime}\right)=t R(q)+t^{\prime} R\left(q^{\prime}\right)$. Hence (b) follows. If $q$ is in $X^{\prime}$ and $C^{\prime}$, then $x$, where $q=L x$, is in $C^{\prime}$ and hence, since $f$ is monotone, $R(q)=f(x)$ is in $K$, and $R$ is monotone.

$\left[Y^{\prime}, X^{\prime}, C^{\prime}, V, K^{\prime}, R, M\right]$ thus satisfy the conditions of Definition 4 and since $\mathfrak{M}$ has the MEP there exists an extension $S$ of $R$ to all of $Y^{\prime}$ with values in $V$ such that $S$ is monotone, distributive and $S(m z)=S(z)$ for every $m$ in $M$ and $z$ in $Y^{\prime}$.

(10) Define $F$ from $Y$ to $V: F(y)=S(L y)$ for all $y$ in $Y$. $F$ is monotone and distributive since both $S$ and $L$ have these properties. $F(x)=S(L x)=f(x)$ for all $x$ in $X$. Hence $F$ is an extension of $f$.

$F(g y)=S(L g y) . L g y(\mathfrak{m})=F^{\prime}\left(g^{\prime} g y\right)$ where $g^{\prime}$ is in the coset $m$. Let $g$ be in the coset $\mathfrak{m}^{*}$. Hence $L g y(\mathfrak{m})=L y\left(\mathfrak{m m}^{*}\right)=m^{*}(L y(\mathfrak{m}))$ for all $\mathfrak{m}$ in $\mathfrak{M}$. Thus $L g y=m^{*} L y$ for all $y$ in $Y$ and $\mathfrak{g}$ in $\mathfrak{m}^{*}$. Therefore $F(g y)=S\left(m^{*} L y\right)=S(L y)$ $=F(y)$ for all $g$ in $G$ and $y$ in $Y$ since $S$ is invariant with respect to elements in $M$. Thus the theorem in proved and $G$ has the MEP.

Given a semigroup with the MEP, it does not necessarily follow that a subsemigroup has the MEP. An example of such a situation will be presented later. However, the statement is true for groups and subgroups.

THEOREM 7. If \&5 is a group with the MEP then any subgroup $\mathfrak{S}$ of \& has the $M E P$.

Proof. The proof of this theorem uses methods similar to the proof of Theorem 6.

(1) Consider $[Y, X, C, V, K, f, H]$ as in Definition 4, where $H$ is a representation of $\mathfrak{S}$. Since $V$ has the LUBP, there exists a monotone distributive extension of $f, F^{\prime}$, to all of $Y$.

(2) Consider the left cosets of $\mathbb{B}$ modulo $\mathfrak{E}, g^{*}$. Pick a representative $\mathfrak{g}$ from each coset $g^{*}$. Thus if $\mathfrak{g}^{\prime}$ is in $g^{*}$ then $\mathfrak{g}^{\prime}=\mathfrak{g} \mathfrak{h}$ for some $\mathfrak{h}$ in $\mathfrak{S}$, where $\mathfrak{g}$ is the representative of $g^{*}$.

(3) Define $A$ to be the vector space of functions from (s) to $V$. Define $L$, a map from $Y$ to $A: L y=z$, where $z\left(\mathfrak{g}^{\prime}\right)=F^{\prime}(h y)$. for every $\mathfrak{g}^{\prime}$ in $B$, where $\mathfrak{g}^{\prime}$ is in $g^{*}, \mathfrak{g}$ is the representative of $g^{*}$, and $\mathfrak{g}^{\prime}=\mathfrak{g h}$. $L$ is well defined and distributive.

Denote by $C^{\prime}$ the image of $C, L C . C^{\prime}$ is a cone and with respect to the 
order determined by $C^{\prime}, L$ is montone. Denote $X^{\prime}=L X$. Again as in the last theorem $z+X^{\prime}$ meets $C^{\prime}$ for every $z$ in $Y^{\prime}$. Also $L x\left(g^{\prime}\right)=f(x)$ for every $x$ in $X$.

(4) Consider the right regular representation $G \#$ of (S) on $Y^{\prime}$. Then it follows that $g \# q=q$ for every $q$ in $X^{\prime}$ and every $g \#$ in $G \#$. Also $g \# z$ is in $C^{\prime}$ for every $z$ in $C^{\prime}$ and $g \#$ in $G \#$.

(5) Define the map $R$ from $X^{\prime}$ to $V: R(L x)=f(x)$ for every $x$ in $X$. It can be shown that $R$ is monotone and distributive, and since $X^{\prime}$ is left point wise invariant by all elements of $G \#, R(g \# q)=R(q)$ for all $q$ in $X^{\prime}$ and $g \#$ in $G \#$. Thus $\left[Y^{\prime}, X^{\prime}, C^{\prime}, V, K, R, G \#\right]$ satisfy the conditions of Definition 4 , and by virtue of the assumption that $\$$ has the MEP, there exists a monotone, distributive extension $S$ of $R$ to all of $Y^{\prime}$ such that $S(g \# z)=S(z)$ for all $g \#$ in $G \#$ and $z$ in $Y^{\prime}$.

(6) Define $F$ from $Y$ to $V: F(y)=S(L y)$ for all $y$ in $Y$. Then exactly as in the previous theorem, $F$ is monotone, distributive and an extension of $f$. It remains to show that $F(h y)=F(y)$ for all $h$ in $H$ and $y$ in $Y$. Since $F(h y)$ $=S(L(h y))$, it will be sufficient to prove that $L(h y)=g \#(L y)$ for some $g \#$ in $G \#$, because $S$ is invariant with respect to each $g \#$ in $G \#$. Now $L(h y)\left(\mathfrak{g}^{\prime}\right)$ $=F^{\prime}\left(h^{\prime} h y\right)$, where $\mathfrak{g}^{\prime}=\mathfrak{g h}^{\prime}, \mathfrak{g}$ is the representative of $g^{*}$ the coset containing $\mathfrak{g}^{\prime}$. Therefore $L h y\left(\mathfrak{g}^{\prime}\right)=L y\left(\mathfrak{g}^{\prime \prime}\right)=L y\left(\mathfrak{g}^{\prime} \mathfrak{g}^{\prime-1} \mathfrak{g}^{\prime \prime}\right)=\left(\left(\mathfrak{g}^{\prime-1} \mathfrak{g}^{\prime \prime}\right) \# L y\right)\left(\mathfrak{g}^{\prime}\right)$, where $\mathfrak{g}^{\prime \prime}$ $=\mathfrak{g} \mathfrak{h}^{\prime} \mathfrak{h}$. Note that $\mathfrak{g}^{\prime-1} \mathfrak{g}^{\prime \prime}=\mathfrak{h}^{\prime-1} \mathfrak{g}^{-1} \mathfrak{g} \mathfrak{h}^{\prime} \mathfrak{h}=\mathfrak{h}$. Hence $\operatorname{Lhy}\left(\mathfrak{g}^{\prime}\right)=(h \# L y)\left(\mathfrak{g}^{\prime}\right)$ for every $\mathfrak{g}^{\prime}$ in (5). That is, $L h y=\mathfrak{h} \#(L y)$, and the theorem is proved.

THEOREM 8. If an abstract group (5) has the MEP, then so has every homomorphic image $\mathfrak{S}$ of $\mathfrak{S}$.

Proof. The proof is trivial and follows from the fact that every representation of $\mathfrak{W}$ is a representation of $\mathfrak{S}$.

From the preceding theorems a few of several obvious corollaries are presented.

COROLlARY 3. If every finitely generated subsemigroup of a semigroup has the $M E P$, then so has the semigroup.

COROLLARY 4. If every finitely generated subsemigroup of a semigroup is solvable, then the semigroup has the MEP.

CoROLlaRY 5. If every finite subset of a group generates a finite subgroup, then the group has the MEP.

The following theorem is concerned with invariant, monotone, distributive projections.

TheOREM 9. Let $Y$ be an OLS with cone $C$. Let $V$ be a subspace of $Y$ such that the induced cone $K$ in $V$ is sharp, and $V$ considered as an OLS has the LUBP. Also assume $y+V$ meets $C$ for all $y$ in $Y$. Let $G$ be a semigroup of operators on $Y$, such that $G$ considered as an abstract semigroup has the $M E P, g y$ is in $C$ for all $g$ in $G$ and $y$ in $C$, and $g v=v$ for all $g$ in $G$ and $v$ in $V$. Then 
there exists a monotone, distributive function $P$ from $Y$ onto $V$ such that $P(v)=v$ for all $v$ in $V$ and $P(g y)=P(y)$ for all $y$ in $Y$ and $g$ in $G$.

Proof. Let $I$ be the identity map on $V$, then $[Y, V, C, V, K, I, G]$ satisfy the conditions of Definition 4, and since $G$ has the MEP, there exists an extension $P$ of $I$ with the desired properties.

Theorem 10. Let $Y, C, V, K$, and $G$ be as in Theorem 9. Let $X$ be an OLS and let $f$ be a monotone, distributive function from $V$ to $X$. Then there exists $a$ monotone, distributive function $F$ from $Y$ to $X$ such that $F$ is an extension of $f$ and $F(g y)=F(y)$ for all $y$ in $Y$ and $g$ in $G$.

Proof. Define $F=f P$, where $P$ is the function considered in Theorem 9. $F$ is clearly the desired extension.

THEOREM 11. Let $V$ be an OLS with the LUBP and whose positive cone $K$ is sharp. Let $X$ be a subspace of $V$ such that $v+X$ meets $K$ for every $v$ in $V$. Let $G$ be a semigroup of operators on $V$ such that $G$ considered as an abstract semigroup has the $M E P, g$ maps $K$ into itself for every $g$ in $G, g x=x$ for every $x$ in $X$ and $g$ in $G$. Then there exists a monotone, distributive operator from $V$ to $V$ such that $F(g v)=F(v)$ for all $v$ in $V$ and all $g$ and $F$ is the identity on $X$.

Proof. Let $I$ be the identity map on $X$. $[V, X, K, V, K, I, G]$ satisfy Definition 4 . Hence the desired extension exists.

4. Invariant means. There is a very close relationship between semigroups with the MEP and the HBEP and semigroups with an invariant mean definable on them. In this section a relationship will be developed and certain counter examples will be presented.

Definition 1. A mean on a semigroup $G$ is a distributive functional $M$ defined on $m(G)$, the linear space of real-valued bounded functions on $G$, such that (a) $M(x) \geqq 0$ for all $x$ in $m(G)$ such that $x(g) \geqq 0$ for all $g$ in $G$,

(b) $M(e)=1$, where $e$ is the constant 1 function on $G$.

It is easily proved and a well known result that condition (a) or (b) in Definition 1 can be replaced by (c) $\|M\|=1$.

Definition 2. Given a semigroup $G$ and $m(G)$, the right (left) regular representation, $R_{o}\left(L_{o}\right)$ on $m(G)$ is defined: $\left(R_{0} x\right)\left(g^{\prime}\right)=x\left(g^{\prime} g\right)\left(\left(L_{0} x\right)\left(g^{\prime}\right)=x\left(g g^{\prime}\right)\right)$ for every $x$ in $m(G)$ and every $g^{\prime}$ in $G$.

Definition 3. A mean $M$ on a semigroup $G$ is right invariant if $M\left(R_{g} x\right)$ $=M(x)$ for every $x$ in $m(G)$ and $g$ in $G$. A similar definition holds for a left invariant mean. A mean is invariant if it is both left and right invariant.

The following theorem which is proved in [6] is quoted. If a semigroup possesses a left invariant mean and a right invariant mean then it possesses an invariant mean. If a group possesses a right (left) invariant mean, then it possesses a left (right) invariant mean and hence an invariant mean.

The collection of semigroups which are known to have invariant means, 
[6], corresponds exactly to the class of semigroups which have been proved here to have the MEP and hence the HBEP. It seems probable that the classes of semigroups with the HBEP, the MEP, and with invariant means correspond. It can be shown that when certain additional restrictions are placed on the range space, then the class of semigroups which have the extension properties with respect to these suitably restricted spaces is precisely the class with invariant means. However, with no additional restrictions the following theorem is easily proved. (A stronger theorem can be proved but requires additional preparatory material.)

THEOREM 1. The class of semigroups with invariant means contains the class with the HBEP (MEP).

Proof. (1) Let $G$ be an abstract semigroup with the HBEP. Consider: $m$, the space of bounded, real-valued functions on $G ; X$, a subspace of $m$ consisting of the constant functions on $G$ (the set of functions of the form te, where $t$ is a real number and $e$ the constant 1 function); $R$, the real numbers.

(2) Define $f$, from $X$ to $R: f(t e)=t$ for every real number $t$. Consider the right regular representation $R_{g}$ on $m$. Since $X$ is left pointwise invariant by this representation, $f$ is trivially invariant with respect to this representation. Define $p$ from $m$ to $R: p(y)=\|y\|$ for every $y$ in $m$. Clearly $f(x) \leqq p(x)$ for every $x$ in $X$. Further since $G g$ is contained in $G, p\left(R_{g} y\right) \leqq p(y)$ for every $y$ in $m$ and $g$ in $G$. The same results hold for the left representation. Hence [ $m, X, R, R^{*}$, $\left.p, f, R_{o}\right]$, where $R^{*}$ is the set of nonnegative real numbers, satisfy the conditions of Definition 4 of the previous section. Since $G$ has the HBEP, there exists a distributive extension $M_{R}$ of $f$ to all of $Y$ which is invariant with respect to the right regular representation, such that $M_{R}(e)=1$, and $M_{R}(y)$ $\leqq\|y\|$ for all $y$ in $Y$. From the last two conditions it follows that $\left\|M_{R}\right\|=1$. Hence $M_{R}$ is a right invariant mean for $G$. Replacing $R_{\theta}$ by $L_{g}$, it follows that there exists a left invariant mean for $G$, hence an invariant mean.

The only examples in the literature of groups which do not possess invariant means and hence do not have the HBEP and the MEP are groups which contain a subgroup which is a free nonabelian group on two generators [6]. An example of a two element semigroup which does not possess an invariant mean is given by the following multiplication rules: $a a=b a=a$, $b b=a b=b$ [6]. An example of a semigroup which possesses the MEP but which contains a subsemigroup which does not is given by adjoining to the above semigroup an element $c$ with multiplication rules: $c c=a c=c a=b c=c b$ $=c$. The proof that this semigroup possesses the MEP is presented.

Consider a representation $\left\{a^{\prime}, b^{\prime}, c^{\prime}\right\}$ on an OLS $Y$ with associated $X, C, V, K, f$, all satisfying the conditions of Definition 4 in $\S 3$. Then since $V$ has the LUBP there exists a monotone, distributive extension $F^{\prime}$ of $f$. Define $F: F(y)=F^{\prime}\left(c^{\prime} y\right)$ for all $y$ in $Y$. This is clearly an invariant monotone distributive extension of $f$. 


\section{BIBLIOGRAPHY}

1. G. P. Akilov, Necessary conditions for the extension of linear operations, Doklady Akad. Nauk SSSR. (N.S.) vol. 59 (1948) pp. 417-418 (Russian).

2. - On the extension of linear operations, Doklady Akad. Nauk SSSR. (N.S.) vol. 57 (1947) pp. 643-646 (Russian).

3. S. Banach, Théorie des opérations linéaires, Warsaw, 1932.

4. G. Birkhoff, Lattice theory, New York, 1948.

5. M. M. Day, Notes on ordered linear spaces, University of Illinois, 1950, unpublished.

6. - Means for the bounded functions and ergodicity of the bounded representations of semigroups, Trans. Amer. Math. Soc. vol. 69 (1950) pp. 276-291.

7. D. B. Goodner, Projections in normed linear spaces, Trans. Amer. Math. Soc. vol. 69 (1950) pp. 89-108.

8. S. Kakutani, Concrete representations of abstract (M)-spaces, Ann. of Math. (2) vol. 42 (1941) pp. 523-537.

9. L. Kantorovič, Linear operations in semi-ordered spaces I, Rec. Math. (Mat. Sbornik) N.S. vol. 7 (1940) pp. 210-279.

10. - - Lineare Halbgeordnete Räume, Rec. Math. (Mat. Sbornik) N.S. vol. 2 (1937) pp. 121-168.

11. - Sur la théorie générale des opérations dans les espaces semi-ordonnés, C. R. (Doklady) Acad. Sci. URSS. N.S. vol. 1 (1936) pp. 283-286.

12. J. L. Kelley, Banach spaces with the extension property, Trans. Amer. Math. Soc. vol. 72 (1952) pp. 323-326.

13. M. G. KreYn, and M. A. Rutman, Linear operators leaving invariant a cone in a Banach space, Uspehi Matematičeskih Nauk N.S. vol. 23 (1948) pp. 3-95. American Mathematical Society Translation No. 26, New York, 1950.

14. A. Morse, and R. Agnew, Extensions of linear functionals, with applications to limits, integrals, measures and densities, Ann. of Math. (2) vol. 39 (1938) pp. 20-30.

15. L. Nachbin, A theorem of the Hahn Banach type for linear transformations, Trans. Amer. Math. Soc. vol. 68 (1950) pp. 28-46.

16. A. Sobczyk, and H. F. Bohnenblust, Extensions of functionals on complex linear spaces, Bull. Amer. Math. Soc. vol. 44 (1938) pp. 91-93.

UNIVERSITY OF IlLINOIS,

URBana, ILl.

ILLINOIS INSTITUTE OF TECHNOLOGY,

Chicago, Ill. 